Revue d'histoire de l'Amérique française

REVUE D.HISTOIRE DE L'AMÉRIQUE FRANÇAISE

\title{
Le raid de la Tortue, le 3 novembre 1838
}

Le « Général » Hubert Lefebvre-Rigoche (1817-1899)

\section{Gaston Derome}

Volume 7, numéro 4, mars 1954

URI : https://id.erudit.org/iderudit/301620ar

DOI : https://doi.org/10.7202/301620ar

Aller au sommaire du numéro

Éditeur(s)

Institut d'histoire de l'Amérique française

ISSN

0035-2357 (imprimé)

1492-1383 (numérique)

Découvrir la revue

Citer cet article

Derome, G. (1954). Le raid de la Tortue, le 3 novembre 1838 : le " Général " Hubert Lefebvre-Rigoche (1817-1899). Revue d'histoire de l'Amérique française, 7(4), 483-489. https://doi.org/10.7202/301620ar d'utilisation que vous pouvez consulter en ligne.

https://apropos.erudit.org/fr/usagers/politique-dutilisation/ 


\section{LE RAID DE LA TORTUE, LE 3 NOVEMBRE 1838}

\section{LE “GENERAL" HUBERT LEFEBVRE-RIGOCHE}

(1817-1899)

Hubert Lefebvre-Rigoche, marchand de La Tortue, fut le chef véritable de l'insurrection dans les paroisses du sud de Laprairie, lors de la reprise des hostilités, en novembre 1838.

Lefebvre-Rigoche fut mis à la tête d'une troupe de 150 hommes, qui prit part à trois engagements: le raid de La Tortue, localite sise à quelque dix milles au sud de Laprairie, le 3 novembre 1838; l'assaut de la demeure du capitaine Frédérick Singer, au village de Saint-Philippe, même date; le combat d'Odelltown, au sud de Lacolle, le 9 suivant.

Lefebvre-Rigoche avait reçu pour mission de s'emparer des casernes de Laprairie et d'incendier le bâteau-traversier, entre Laprairie et Montréal, le Princess Victoria.

Arme au poing, il avait obligé Joseph Robert, capitaine de milice démissionnaire, de commander la troupe qui marcha sur Laprairie ${ }^{2}$.

Le parti de Patriotes fit d'abord halte chez l'aubergiste de La Tortue, Eugène Rousseau, qu'il fit prisonnier ${ }^{3}$, puis continua sa route en direction de Laprairie, non sans prendre soin de désarmer

1. Rendant témoignage le 8 janvier 1839 devant le Conseil de guerre, présidé par le major John Clitherow, Florence Longtin donne le qualificatif de "Général" à Lefebvre-Rigoche, en spécifiant que tout le monde le désignait sous ce nom. - V. Report of the State Trials, (Montréal, 1839), 1: 265.

Devant le même tribunal et à la même date, un cultivateur de La Tortue, Cyprien Boyer déclare que "Hubert Lefebvre-Rigoche était armé d'un sabre, d'un pistolet et d'une carabine et qu'il menaçait ceux qui ne feraient pas leur devoir, de leur tirer une balle dans le corps". Op. cit., 267.

2. Deposition de Joseph Robert, le 19 novembre 1838, Archives de la Province de Québec.

3. Report of the State Trials, op. cit., 262. 
les Loyalistes qu'il rencontrait en chemin. Au début, cette opération se fit sans trop de difficulté, mais, rendu à la maison de David Vitty où s'étaient réunis les Loyalistes, on menaça d'enfoncer la porte. A un coup de feu, tiré de l'intérieur de la maison ${ }^{4}$, les Patriotes ripostèrent par une fusillade. Résultat, le propriétaire, Vitty, fut grièvement blessé et l'un des réfugiés, Aaron Walker, fut tué instantanément.

Après cette malheureuse affaire, le capitaine Robert, suivi d'une cinquantaine d'hommes, traversa la rivière La Tortue et alla rejoindre le détachement de Saint-Constant, levé par Médard Hébert, notaire, de Laprairie. De là, Robert se rendit à Napierville et "après une journée de repos, dit-il, nous continuâmes notre route sur Odelltown... Après avoir été repoussés, nous retraitâmes aux Cồtes [ Napierville ]... et le lendemain, chacun se retira chez lui..."

Après l'attaque de la maison Vitty, Lefebvre-Rigoche réorganisa son bataillon et partit, avec environ 150 hommes, en direction du village de Saint-Philippe où il fit le siège de la maison du capitaine chouayen Frédérick Singer. Au témoignage de Toussaint Legrand", Lefebvre-Rigoche "donna l'ordre de faire feu... plusieurs coups furent tirés sur la maison..."

Heureusement, il n'y eut pas de perte de vie, ni de blessés a cet assaut.

\section{Au Camp de Napierville}

Après cette deuxième fusillade, le capitaine François Surprenant-Lafontaine (1789-1859), présent à cet engagement, qui avait été très actif dans le recrutement des Patriotes à Saint-Philippe, donna l'ordre de se rendre à La Tortue. Il fut plus tard, l'un des 98 condamnés à mort, mais fut gracié. C'est alors que Lefebvre-Rigoche prit le commandement. On se mit en route et à mi-chemin de La Tortue, Lefebvre-Rigoche et sa troupe prirent le déjeûner chez François Lefebvre. "Il était 7 heures du matin (4 novembre), dit

4. Ibid..., 237.

5. Déclaration de Toussaint Legrand-Dufresne, 27 novembre 1838, Archives de la Province de Québec. 
Amable Vadboncœur ${ }^{6} .$. ils partirent environ 200 hommes pour La Tortue et arrêtèrent chez François Lefebvre où ils prirent le déjeûner, sous les ordres de Hubert Lefebvre qui avait pris le commandement..."

Rendus à La Tortue, Lefebvre-Rigoche et son attroupement se joignirent, eux aussi, au détachement de Saint-Constant, commandé par Médard Hébert. On tint conseil de guerre. On décida de ne pas pousser à l'attaque projetée de Laprairie, et les deux troupes, quelques jours plus tard, rallièrent le camp de Napierville où près de 4000 hommes étaient assemblés ${ }^{6^{\mathrm{a}}}$ à un moment donné.

\section{A Odelltown}

Le 9 novembre suivant, Hubert Lefebvre-Rigoche, avec Hippolyte Lanctot, notaire ${ }^{7}$, de Saint-Remi, François Camyré, de SaintConstant, fut l'un des principaux officiers sous le major Médard Hébert, qui commandait la colonne du centre au combat d'Odelltown, comme en témoigne un autre capitaine chouayen, Michel Lussier ${ }^{8}$, de Saint-Édouard-de-Napierville.

- "Il (Lussier) vit, dit-il, Hippolyte Lanctot à cheval sur une jument qui - lui appartenait... Médard Hébert lui enleva aussi un cheval, une charrette puis - un harnois... Hébert, Lanctot, Lefebvre Hubert, Desmarais Abraham, étaient armés de sabres et d'épées..."

Après le combat d'Odelltown, Hubert Lefebvre-Rigoche, chef de la troupe de La Tortue, eut la sagesse de mettre la frontière entre lui et les sbires de Colborne et de fuir aux États-Unis.

6. Déclaration d'A mable Vadboncœur, 18 novembre 1838, Archives de la Province de Québec. Amable Vadboncœur, arrêté le 19 novembre 1838, était fils d'Amable Vadboncœur et de Clotilde Desmarais; il épousa en 1839 à Saint-Philippe, Émélie Tallard. Le patriote Amable Vadboncœur mourut nonagénaire, le 26 octobre 1908 a Saint-Michel-de-Napierville. (La Presse, 29 octobre 1908).

6a. Report of the State Trials... op. cit., 124.

7. Hippolyte Lanctot (1816-1887), notaire, a exercé sa profession à SaintRemi, à Saint-Edouard, à Laprairie et à Montréal; il s'occupa de politique et brigua deux fois les suffrages de l'électorat, dans Huntingdon-Laprairie en 1849 et dans Laprairie en 1857. V. Les Députes de La Prairie, - Centenaire de Saint-Isidore-deLaprairie (Montréal, 1934).

8. Déclaration de Michel Lussier, de Saint-Édouard, 23 novembre 1838, Archives de la Province de Québec. 
Pendant ce temps, neuf membres de son parti, soit le capitaine Joseph Robert ${ }^{9}$, Jacques Robert ${ }^{10}$ - pas un proche du précédent les deux frères, Ambroise et Charles Sanguinet ${ }^{11}$, fils de l'ancien seigneur de La Salle ${ }^{12}$, Pascal Pinsonneau ${ }^{13}$, François-Xavier Hamelin dit le "Petit Hamelin" - cousin de Lefebvre-Rigoche - Théophile Robert, Joseph Longtin et Jacques Longtin ${ }^{14}$ eurent à répondre de la mort de Aaron Walker, en janvier 1839, devant le Conseil de guerre qui avait déjà jugé en décembre précédent, le député de Laprairie, Joseph-Narcisse Cardinal (1808-1838), son clerc, Joseph Duquette (1815-1838) et al ${ }^{15}$.

Quatre d'entre eux, Joseph Robert, les frères Sanguinet et le "Petit Hamelin", tous de La Tortue, montèrent sur l'échafaud où les avaient précédés, un mois plus tôt, Cardinal et Duquette ${ }^{16}$. Il n'y a pas d'hésitation à dire que pris, Lefebvre-Rigoche eut été du nombre.

\section{État civil de Leffebvre-Rigoche}

Pour les notes d'identité, j'ai eu recours à $M$. Jean-Jacques Lefebvre, archiviste du palais de justice de Montréal.

Hubert Lefebvre-Rigoche naquit à La Tortue le 28 octobre 1817 et fut baptisé à Saint-Philippe-de-Laprairie. Il était fils de Benoît Lefebvre (1788-1823), maître-forgeron, mort prématurément.

Le plus jeune frère de son père, Basile Lefebvre-Rigoche (18051880) fut élu le premier maire de Saint-Remi-de-Napierville en 1845.

9. V. "Le capitaine Joseph Robert", par M. Jean-Jacques Lefebvre, La Presse, (Montréal, 24 juin 1933).

10. Jacques Robert, né à Saint-Philippe en 1785, y épousa en 1810 Clémence Trudeau; il convola en 1836 à Saint-Édouard avec Josephte Pinsonneau; il mourut à Saint-Edouard le 16 août 1849, victime de l'épidémie du choléra.

11. Emile Falardeau, BRH, avril 1927.

12. Sur les Sanguinet, v. M. Jean-Jacques Lefebvre, BRH, avril 1927 et decembre 1948, aussi Mémoires de la S.G.C.F., (janvier 1946).

13. BRH, février 1947 .

14. Jacques Longtin (178-1869), de Saint-Constant, fut le protégé de Mère Gamelin à la prison de Montréal.

15. L.-O. David, Les Patriotes de 1837-1898 (Montréal, 1884), 199.

16. Aegidius Fauteux, Patriotes de 1897-1898 (Montréal, 1950), 362. 
Son surnom de Rigoche provenait du prénom de son aieul paternel, Ignace-Rigobert Lefebvre (1758-1834), lequel avait épousé à Boucherville, en 1780, sa cousine, Isabelle Sentenne (17611834), fille d'un sergent du Royal Américain, John Santon - nom francisé plus tard en Sentenne ${ }^{17}$ - et de Charlotte Lefebvre (17261791).

Enfin, sa mère, Catherine Vaschereau-Versailles (1789-1859), sœur de la mère du malheureux "Petit Hamelin", convola en 1824 avec Louis Sédilot-Montreuil. De ce second mariage, elle fut l'aleule, entre autres, de notre contemporain, Wilfrid Cédilot (1862-1940), qui a été le dernier député de Laprairie à l'Assemblée Législative de Québec, de 1916 à 1923, avant la fusion des collèges électoraux de Napierville et de Laprairie.

Hubert Lefebvre-Rigoche reçut son éducation à l'École de langues classiques que tenait au village de Saint-Philippe, le curé Pigeon $^{18}$, un homme d'initiative, qui publia un journal, dans son village, en 1826 .

Bénéficiant de l'amnistie, Hubert Lefebvre-Rigoche revint de l'exil et épousa aussitôt (1844), en son village natal de Saint-Philippede-Laprairie, Adelaỉde Tremblay (1820-1872), tante paternelle, entre autres, d'Ernest Tremblay (1852-1904), le grand journaliste dont Aegidius Fauteux a parlé dans son Courrier historique et littéraire ${ }^{19}$. Elle mourut à Montréal en 1872.

Il avait eu six enfants dont trois devenus adultes, tous nés à Saint-Philippe-de-Laprairie: Lucien, né en 1848, Joséphine, née en 1854 et Rosalie en 1856.

Notons les parrains des enfants de Hubert Lefebvre-Rigoche: Son beau-frère, Julien Tremblay, qui fut le père du docteur A.-L. Tremblay (1846-1879), co-fondateur du premier journal francoaméricain avec Ferdinand Gagnon; François-Xavier Bonneau (1808-1895), plus tard capitaine de milice, marchand à La Tortue pendant plusieurs années, dont une fille, Justine Bonneau, religieuse

17. C. Tanguay, Dictionnaire généalogique... VII: 169. - "En marge de trois siècles d'histoire domestique", Revue de l'Université d'Ottawa (1947).

18. "Saint-Constant et Saint-Philippe-de-Laprairie", La Societe Canadienne de l'histoire de l'Église catholique (rapport 1945-46), 140-143.

19. La Patrie, Montréal, 16 février 1936. 
hospitalière, décédée en 1898, fut supérieure de l'Hôtel-Dieu de Montréal, et enfin, sa belle-sœur, Zoé Tremblay-Lanctot, qui fut la mère du juge Husmer Lanctot, et du docteur Joseph Lanctot, conseiller législatif.

Hubert Lefebvre-Rigoche avait deux frères: son aîné, Joseph, né en 1810, marié en 1832 à Pauline Chatel, qui vécut longtemps à Saint-Édouard-de-Napierville et demeurait en 1890 à Saint-Albert de Russell, Ontario, et un frère cadet, Olivier-L. (1822-1903) qui épousa à Saint-Philippe en 1844 Mathilde Deneau (1822-1903), fille de Charles Deneau, voyageur au Nord-Ouest.

Olivier avait géré les affaires de son frère pendant l'exil de celuici, mais au retour, ils vinrent en difficulté et pour régler et éviter un procès, ils prirent un accord devant notaire. ${ }^{20}$

Hubert Lefebvre fut un temps instituteur à Saint-Philippe, probablement à La Tortue où il possédait une assez grande ferme. Le 26 août 1862, devant A. Beauvais, notaire ${ }^{21}$, Hubert LefebvreRigoche cédait à François Riel-Irlande, une terre sise à La Tortue, dans Saint-Philippe, de $5 \times 30$ arpents, tenant par devant à la rivière La Tortue, d'un côté à son frère, Olivier... et qu'il possède par "bons titres" pour la somme de 42,000 livres (anciens cours).

La maison, construite vers 1805 par son père le forgeron Benoît Lefebvre, fut démolie en 1914. Elle fut réquisitionnée par les autorités militaires pour loger un peloton de soldats d'un régiment de Glengarry, qui faisait durant les jours sombres de novembre 1838, l'occupation de la région de La Tortue, alors en ébullution.

Je le consigne ici pour mes fils quand ils auront l'âge de s'intéresser à ces choses, cette ferme passa en 1895 à mon père, Gustave Derome $(1871-1940)$, qui y éleva sa famille.

Après la mort de sa femme, lors de la crise économique de 1872, Hubert Lefebvre-Rigoche partit pour les Etats-Unis avec son fils, Lucien et ses deux filles. Ces deux dernières vécurent dans l'État du Michigan. L'une, Joséphine, avait épousé Pierre Lalonde, l'autre, Rosalie, était mariée à Edmond Lemieux. Hubert Lefebvre-Rigoche alla mourir en octobre 1899 à Minneapolis, Minnesota, où son fils,

20. Archives judiciaires de Montréal.

21. Archives judiciaires de MontrEal. 
Lucien, qui était marié à une demoiselle Colin, s'éteignit lui-même en 1933.

Une petite-fille de Hubert Lefebvre-Rigoche, madame Clotilde Lefebvre-Schwartz, de Minneapolis, est venue par deux fois à Montréal en 1945 et 1946 et m'a procuré la photographie de son grandpère.

Ainsi mourut sur la terre d'exil le Patriote Hubert LefebvreRigoche, qui à peine majeur, en 1838 , avait réussi à conduire 150 hommes dans une insurrection armée contre le plus puissant Empire de l'époque et fut indirectement la cause de la mort sur l'échafaud de quatre de ses coparoissiens et de la ruire et de l'exil pour tant d'autres.

Montréal, décembre 1953.

Gaston Derome

AVIS - LA REVUE D'HISTOIRE DE L'AMERIQUE FRANÇAISE vit de ses abonnements, et rien que de ses abonnements. Elle compte que vous renouvellerez le vôtre, dès ces jours-ci, pour ne pas manquer le no de juin prochain, ler de la 8ème année. 